Chinese Journal of Organic Chemistry

\title{
中环大环化合物合成研究进展
}

张馨元 林 礼李 静段世妤 隆宇航 李加洪*

(西南交通大学生命科学与工程学院 成都 610031)

\begin{abstract}
摘要 中环 $(8 \sim 11$ 元环)与大环(12 元环及以上)是药物和天然产物中非常重要的结构并广泛应用于药物合成、有机化学 以及其他领域. 科研工作者一直致力于开发更加简单、绿色、高效的合成中环大环的方法. 归纳并总结近五年来最新 的通过扩环和环化反应方法合成中环大环化合物的研究现状和进展，并对其未来的发展方向进行了总结和展望.
\end{abstract}

关键词 中环化合物; 大环化合物; 扩环反应; 环化反应

\section{Recent Progress in the Synthesis of Medium-Sized Ring and Macrocyclic Compounds}

\author{
Zhang, Xinyuan Lin, Li Li, Jing Duan, Shiyu Long, Yuhang Li, Jiahong* \\ (School of Life Science and Engineering, Southwest Jiaotong University, Chengdu 610031)
}

\begin{abstract}
Medium-sized rings ( $8 \sim 11$ membered ring) and macrocycles (12-membered rings and above) are extremely important parts of drug and natural products. Meanwhile, they are widely used in the field of medicinal chemistry, organic chemistry and other fields. Developing simple, green and efficient protocol to synthesize medium-sized rings and macrocycles has attracted great interests from chemists in the recent years. The latest ring expansion/cyclization reaction in the synthesis of medium-sized ring and macrocyclic compounds in the past five years is reviewed, and the prospect of their future and development is also outlined.
\end{abstract}

Keywords medium-sized ring; macrocycle; ring expansion reaction; cyclization

中环化合物为 $8 \sim 11$ 元环化合物, 大环化合物为含 有 12 元环及以上的化合物 ${ }^{[1]}$. 它们大量存在于自然界中 的天然产物以及可以针对各种生物靶点的具有重要医 学意义的化合物中, 它们是许多具有生物活性的天然产 物的结构核心 ${ }^{[2]}$. 与相应的线性结构相比, 中环大环提 供的构象约束可以增强结合亲和力 ${ }^{[3]}$, 改变生物利用度, 增强细胞通透性 ${ }^{[4]}$, 展现出具有吸引力的特性和应用价 值.

中环和大环化合物虽具有广阔的应用前景, 但由于 不利的跨环相互作用和熵效应, 中环的环化反应通常遭 受分子间反应的影响 ${ }^{[5]}$, 很难用传统的环化方法构建与 制备. 目前为止, 几乎没有通用的合成方法, 高效合成 这类化合物仍然是一个挑战. 研究简便、高效、环保的 构建中环大环化合物的方法仍是具有强吸引力和挑战 性的前沿课题 ${ }^{[6]}$. 鉴于中环大环的重要性和应用性, 学 术界和制药产业一直在努力开发高效的合成路线 ${ }^{[2 a]}$. 近
年来中环大环化合物的合成方法发展迅速, 取得了重要 的研究进展，如金属催化 ${ }^{[7]}$ 及烯烃复合反应 ${ }^{[5]}$ 等方法. 近年来，从简单易得的原料出发，新的合成路线和构建 策略不断涌现, 因此有必要对新方法、新成果进行总结 归纳. 考虑到烯烃复合反应方法已有许多综述 ${ }^{[5,8]}$, 因此 本文将不再对这类方法进行赘述. 由于含有大环的生物 大分子种类多且结构复杂, 我们也不再对生物大分子的 合成进行综述. 本文结合近五年国内外研究报道, 按照 环化反应和扩环两个方面进行分类, 对合成中环大环的 新试剂、新方法的进展以及机理进行归纳和总结, 希望 对该领域的发展起到一定的促进作用.

\section{1 环化反应}

\section{1 多组分环化反应}

多组分反应(MCR) 可用于大环化合物的合成 ${ }^{[9]}$. 2016 年, Dömling 课题组 ${ }^{[9]}$ 首次报道了能够封闭大环的

* Corresponding author. E-mail: jiahongljh@163.com

Received October 18, 2020; revised November 30, 2020; published online December 9, 2020.

Project supported by the Student's Platform for Innovation and Entrepreneurship Training Program (No. 201910613081)

大学生创新创业(No. 201910613081)资助项目. 
Passerini 反应. 该方法通过一系列 Ugi 多组分反应, 然 后利用能够封闭大环的分子内 Passerini 多组分反应人 工合成具有 15 20 元环的二肽(Scheme 1). 2017 年, 该 课题组还报道了通过 Ugi-MCR 合成中环 ${ }^{[10]}$ 和大环 ${ }^{[11]}$ 的 $^{-}$ 方法. 利用环状羧酸酸酐和二胺反应开环生成 $\alpha, \omega$-氨基 酸, 随后 $\alpha, \omega$-氨基酸、羰基组分和异腈发生 Ugi 反应实 现闭环, 生成含有 8 19 元环的化合物(Scheme 2).

\section{2 重排环化反应}

2019 年, 彭谦、朱守非与叶龙武等 ${ }^{[12]}$ 报道了一种以 Brønsted 酸(BA)为催化剂, 炔酰胺 $\mathbf{1}$ 在 $80{ }^{\circ} \mathrm{C}$ 的 $\mathrm{PhCl}$ 中 反应 $4 \mathrm{~h}$, 合成 8 元苯并 $[d]$ 偶氮酮 2 的反应. 基于密度泛 函理论(DFT)计算、动力学热力学计算以及过渡态检测 等方法, 作者提出了反应机制: 羟基与 HOTf 活化的酰 胺 $\mathbf{1}$ 反应, 生成中间体 $\mathbf{A}$ 后转化为氧鎓过渡态 $\mathbf{B}$, 当使 用手性 Brønsted 酸时, 所得中间产物 $\mathbf{A}^{\prime}$ 分子内发生离子
配对和氢键相互作用, 生成热力学稳定的 $E$ 型中间产物 C, C 发生立体特异性[1,3]-重排生成含有 8 元环的 2 (Scheme 3).

\section{3 级联环化反应}

2019年, Unsworth 课题组 ${ }^{[13]}$ 报道了一个以线性分子 3 为底物, 合成含氮中环的环化/扩环级联反应. 在室温 条件下, 以丙烷膦酸酸䣶(T3P) 和 $\operatorname{NEt}(i-\operatorname{Pr})_{2}$ 为催化剂, 在 $\mathrm{CHCl}_{3}$ 中搅拌 $30 \mathrm{~min}, \mathbf{3}$ 中的吡啶环中的碳氮键与羧 基会酰化生成 $N$-酰亚胺离子，随后分子内发生环化反 应生成中间体 D, D 发生分子内重排, $\mathrm{C}-\mathrm{N}$ 键断裂导 致扩环获得 10 元环 4 (Scheme 4).

\section{4 金属催化环化反应}

2015 年, Igawa 和 Tomooka 课题组 ${ }^{[14]}$ 报道了通过来 源于钴络合物 5a 的双核 2-丁炔单元 5 , 与双(杂)取代非 环单元 6 发生 Double Nicholas 反应, 有效生成中型环炔

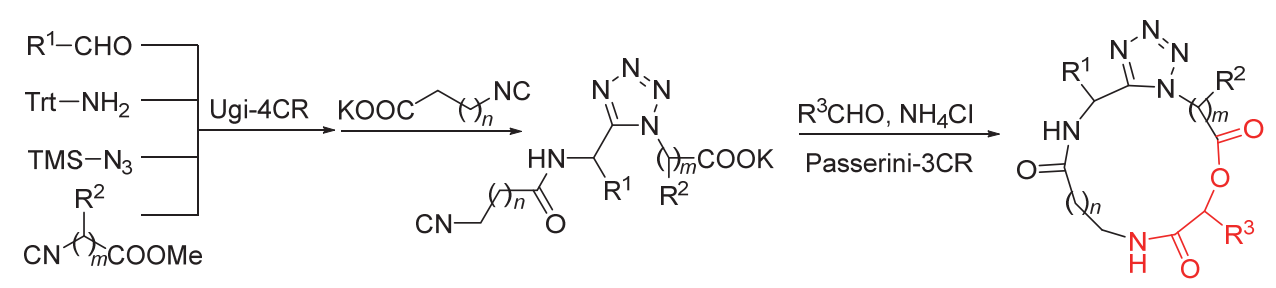

图式 1 大环二肽的合成策略

Scheme 1 Synthesis strategy toward macrocyclic depsipeptides

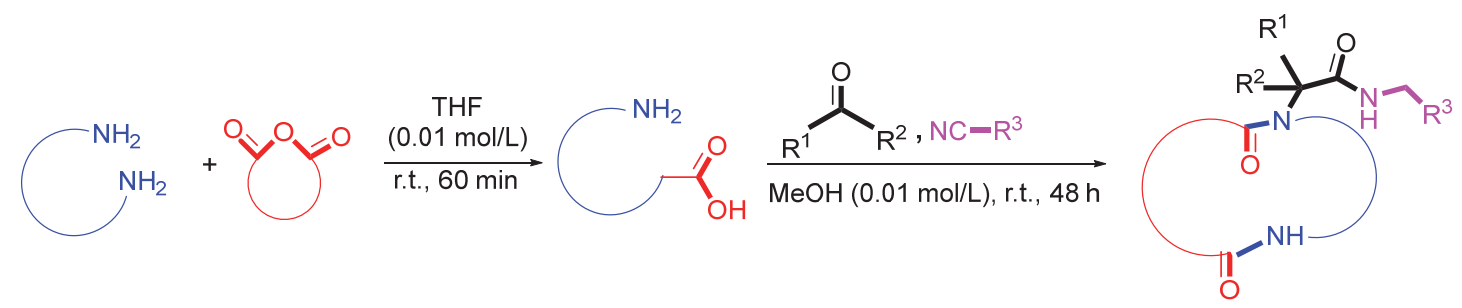

图式 2 Ugi 多组分反应

Scheme 2 Ugi multicomponent reaction

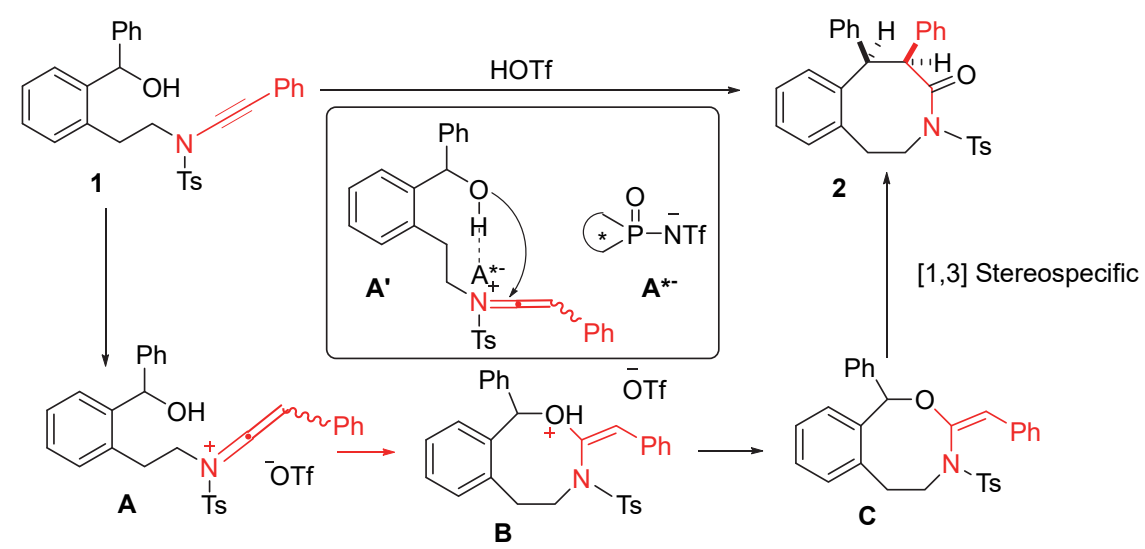

图式 3 氢烷氧化/[1,3]-重排反应

Scheme 3 Hydroalkoxylation/stereospecific [1,3]-rearrangement 


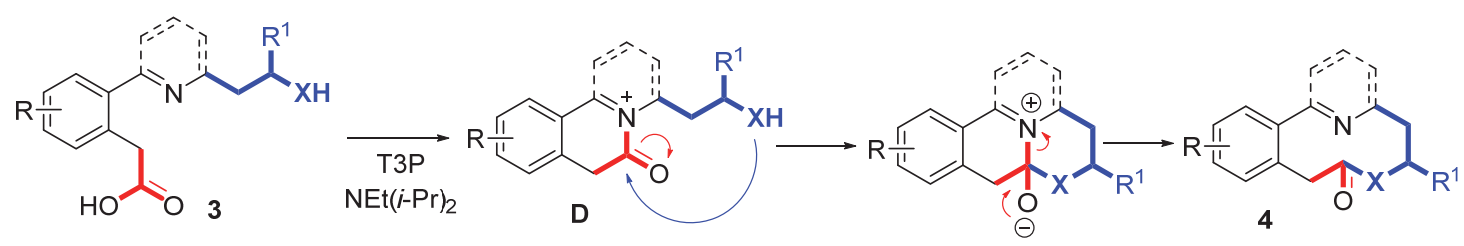

图式 4 环化/扩环级联反应

Scheme 4 Cyclisation/ring expansion cascade synthesis

的方法(Eq. 1). 以 $\mathrm{BF}_{3} \cdot \mathrm{OEt}_{2}$ 为催化剂, 室温条件下, $\mathbf{5 b}$ 或 $5 \mathbf{c}$ 与 $6 \mathbf{a}$ 反应产生的中环 $(X, Y=N T s)$, 在大量硅胶存 在下用硝酸铈铵 $(\mathrm{CAN})$ 处理, 可高效地获得 $8 \sim 11$ 元环 7 (Scheme 5).
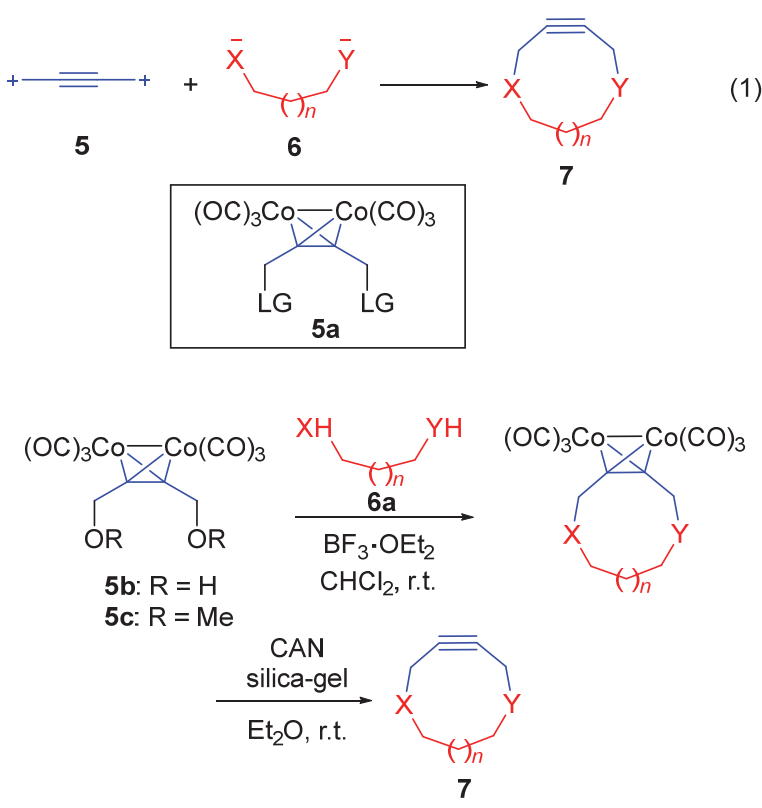

图式 5 Double Nicholas 反应

Scheme 5 Double Nicholas reaction

2018 年, Chan 课题组 ${ }^{[15]}$ 报道了以 $\mathrm{Au}^{\mathrm{I}}$ 为催化剂, 1烯-4,10-二炔酯为底物, 生成含有 8 元环的双环化合物 的反应(Scheme 6). 底物 8 生成的中间态 9 发生 Rautenstrauch 重排生成 10, 同时炔基与金属催化剂配位, $\mathrm{Au}^{\mathrm{I}}$ 激活 10 发生 1,5 -氢转移/8-内插入环化, 生成含有 8 元环 的双环产物 11.

\section{2 扩环反应}

\section{1 重排扩环反应}

2015 年, Unsworth 课题组 ${ }^{16]}$ 报道了一种能够连续扩 环的 SuRE 反应(Successive ring-expansion). 该反应以环 状 $\beta$-酮酸酯为底物, 最终得到含有 $9 \sim 24$ 元的环内酰胺 和内酯. 将环状 $\beta$-酮酸酯 $12 、 \mathrm{MgCl}_{2}$ 和吡啶在 $\mathrm{CH}_{2} \mathrm{Cl}_{2}$ 中混合 $30 \mathrm{~min}$, 然后加入酰氯并在室温下搅拌 $1 \sim 2 \mathrm{~h}$, 生成中间产物 13 , 再在反应中加入吡啶, 生成扩环产物

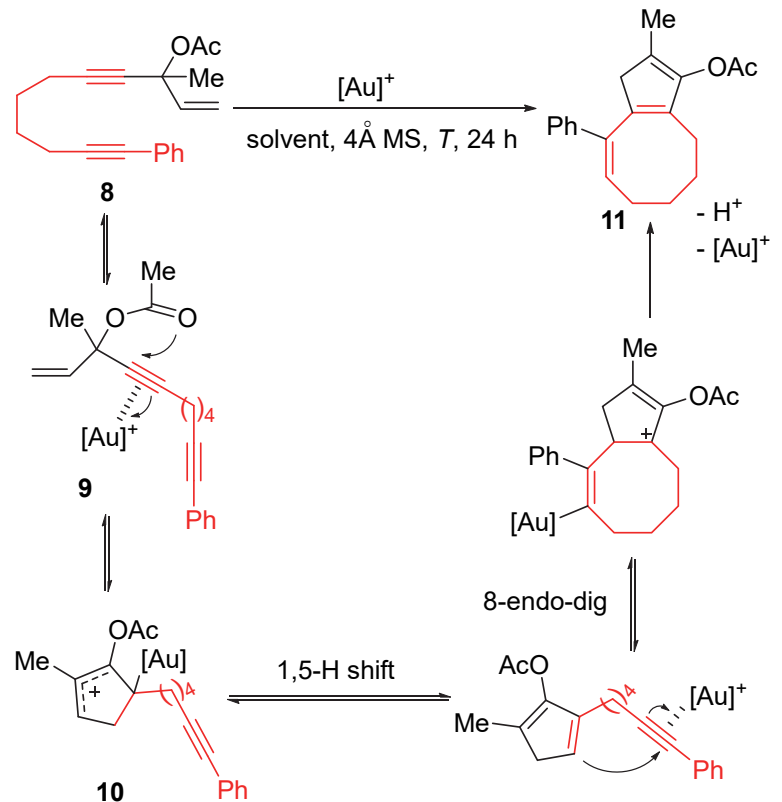

图式 $6 \mathrm{Au}^{\mathrm{I}}$ 催化 1-烯-4,10-二炔酯的反应

Scheme $6 \mathrm{Au}^{\mathrm{I}}$-catalyzed reactivities of 1-ene-4,10-diynyl esters

14 (Scheme 7). 该反应的机理为: 环状化合物 15 与线性 片段 16 偶联后发生重排, 并发生扩环反应形成产物 17. 17 可以与线性片段重复相同的过程实现连续扩环 (Scheme 8).

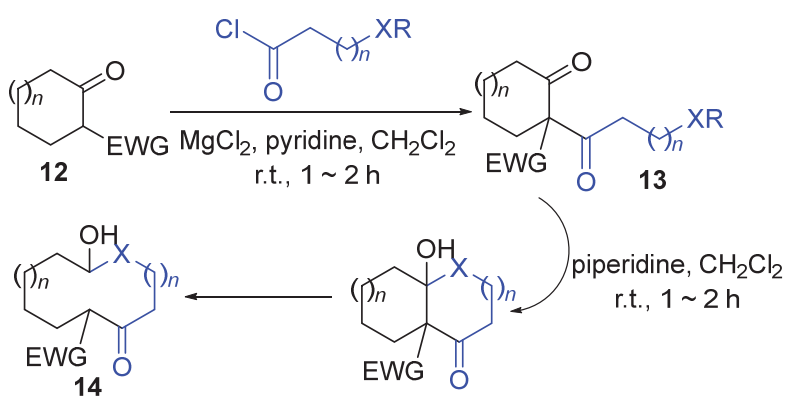

图式 7 环状 $\beta$-酮酸酯 SuRE 反应

Scheme 7 SuRE reaction of cyclic $\beta$-ketoester

2017 年, Unsworth 课题组 ${ }^{[17]}$ 报道了内酰胺连续参与 并生成 8 25 元环的 SuRE 反应(Scheme 9). 2018 年, 该 课题组 ${ }^{[18]}$ 又报道了新的 SuRE 方法. 该方法以羟基酸 


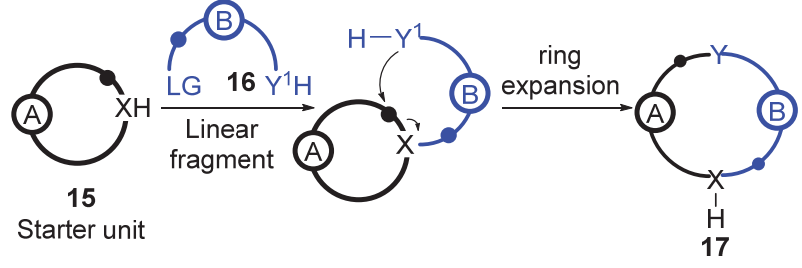

图式 8 SuRE 反应

Scheme 8 Successive ring-expansion (SuRE)

为底物, 连续与内酰胺反应, 生成含有 $10 \sim 24$ 元环的内 酯或内酰胺(Scheme 10).

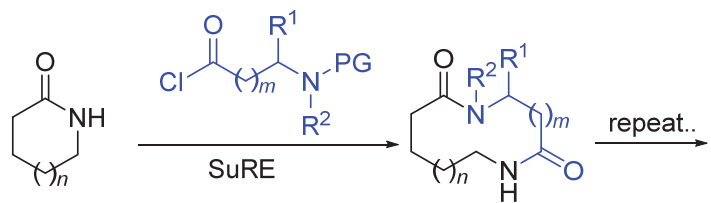

图式 9 内酰胺 SuRE 反应

Scheme 9 SuRE reaction of lactam

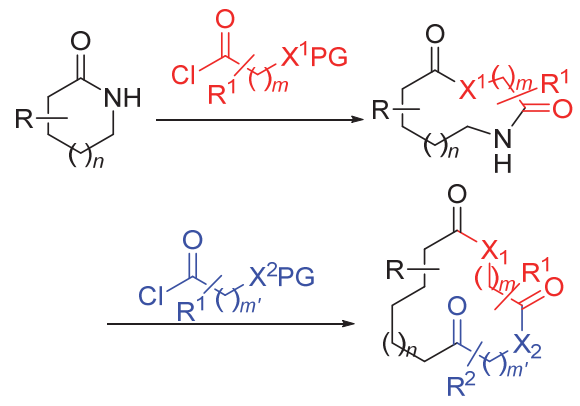

图式 10 大环内酯 $\mathrm{SuRE}$ 反应

Scheme 10 SuRE reaction of macrolide

2017 年, Clayden 课题组 ${ }^{[19]}$ 报道了以邻胺基苯甲酰 胺 20 为底物, 利用 Smiles 重排进行 $n \rightarrow n+4$ 扩环的反 应. 在碱环境下, 靛红酸酐类化合物 18 与含氮杂环 19 在四氢呋喃(THF)中混合可获得 20.20 在回流的 THF 中, 在 NaHMDS 作用下发生重排, 获得含有 $9 \sim 12$ 元环的扩 环产物 21 (Scheme 11).

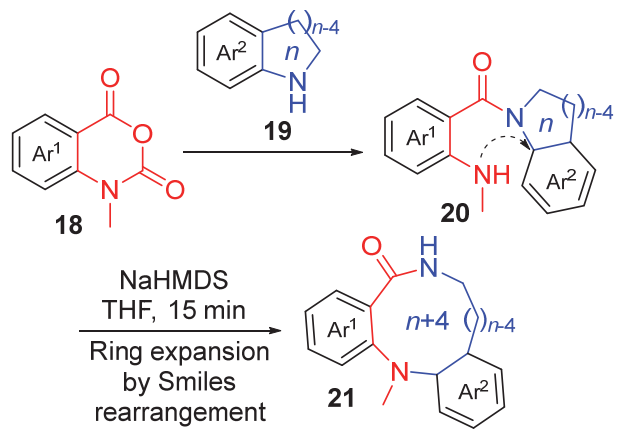

图式 11 Smiles 重排扩环反应

Scheme 11 Smiles rearrangement ring expansion reaction

\section{2 基于环张力的扩环反应}

2015 年, 李子刚和孙建伟课题组 ${ }^{[20]}$ 报道了以 $N$-磺 酰基亚胺和甲硅烷氧基炔为底物合成中环内酰胺的反 应. 其机理为: $N$-磺酰亚胺 $\mathbf{2 2}$ 在酸的催化作用下脱去水 或醇并被活化后与甲硅烷氧基炔烃 $\mathbf{2 3}$ 反应生成中间体 $\mathbf{E}, \mathbf{E}$ 中的 $\mathrm{C}-\mathrm{N}$ 键断裂并释放环张力生成含有 7 8 元 环的扩环产物 24 (Scheme 12).

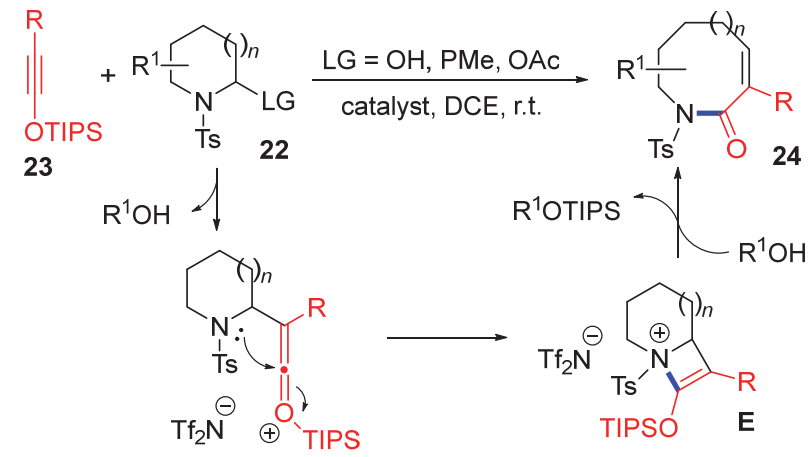

图式 12 环状半缩醛的催化扩环反应

Scheme 12 Catalytic ring expansion of cyclic hemiaminals

2019 年, Coquerel 和 Rodriguez 等 ${ }^{[21]}$ 报道了在双功 能氨基催化剂作用下，基于 Michael 加成反应的级联扩 环反应. 其机理为：以 $N$-芳基仲酰胺基活化的环丁酮 $\mathbf{2 5}$ 和邻氨基硝基苯乙烯衍生物 26 作为底物, 在双功能氨 基催化剂作用下，发生 Michael 加成生成 F, F 环化生成 $\mathbf{G}$ ，随后键断裂并释放环张力形成含有八元环的 27 (Scheme 13).

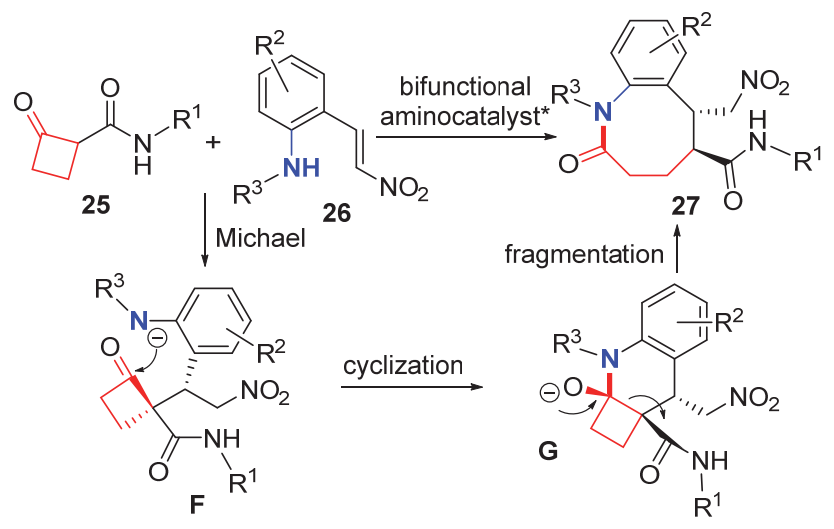

图式 13 Michael 加成/扩环级联反应

Scheme 13 Michael addition and ring expansion cascade

\section{3 环加成扩环反应}

2014 年, Krische 课题组 ${ }^{[2]}$ 报道了以钓(0)作催化剂, 以二醇与二烯为底物, 发生 $[4+2]$ 环加成生成中环的方 法. 环烷二醇 28 和 1,3-二烯 29 可以反应获得 30. 在室 温条件下, 环加成产物 30 在亚碘酰苯双乙酸和 $\mathrm{PhI}(\mathrm{OAc})_{2}$ 作用下，[n.4.0]环发生氧化裂解，生成含有 
$9 \sim 12$ 元环的 31 (Scheme 14).

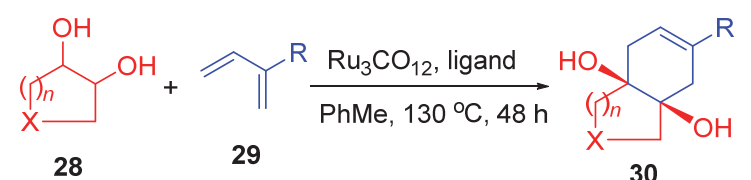

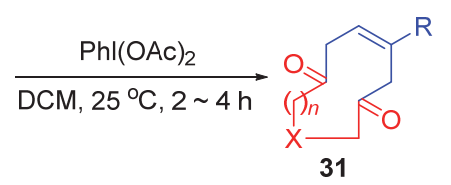

图式 14 钉(0)催化二醇二烯 $[4+2]$ 环加成

Scheme 14 Ruthenium $(0)$ catalyzed diol-diene [4+2] cycloaddition

2017 年, Zhao 等 ${ }^{[23]}$ 报道了以 $\mathrm{Pd}$ 作催化剂, $N$-甲苯磺 酰基氮杂二烯与丁二烯碳酸酯为底物, 生成 9 元杂环的 $[5+4]$ 环加成反应. 作者认为其机理为: 35 与 36 反应生 成中间体 $\mathbf{H}$, 随后形成 $\mathbf{I}, \mathbf{3 6}$ 的空间位阻导致反应区域发 生变化, 最终发生 $[5+4]$ 环加成反应形成 9 元杂环 37 (Scheme 15). 2018 年, Zhao 等[24]报道了在以 $\operatorname{Pd}(\mathrm{dba})_{3} \bullet$ $\mathrm{CHCl}_{3}$ 作催化剂, 膦配体作用下, 乙烯基氧烷与氮杂二 烯在 $40{ }^{\circ} \mathrm{C}$ 的 $\mathrm{CH}_{2} \mathrm{Cl}_{2}$ 溶液中反应 $19 \mathrm{~h}$, 得到 10 元杂环 的 $[6+4]$ 环加成反应(Eq. 2). 该方法也可以合成九元杂 环化合物(Eq. 3).<smiles>[R]C=C1Oc2ccccc2C1=[N+]=[N-]</smiles>

35

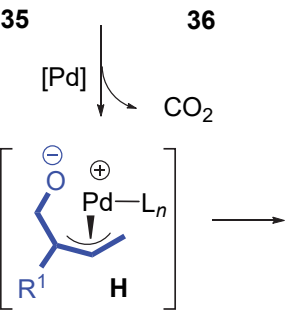

$[\mathrm{Pd}]$<smiles>[R]C1=CCN([123I])c2oc3ccc(F)cc3c2C([R])OC1</smiles>

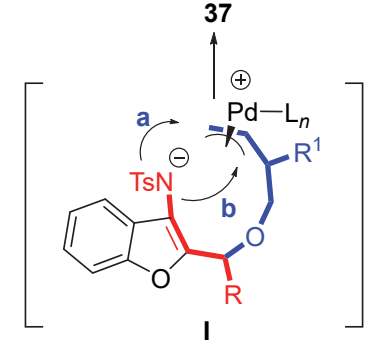

图式 $15[5+4]$ 环加成反应

Scheme 15 [5+4] Cycloaddition

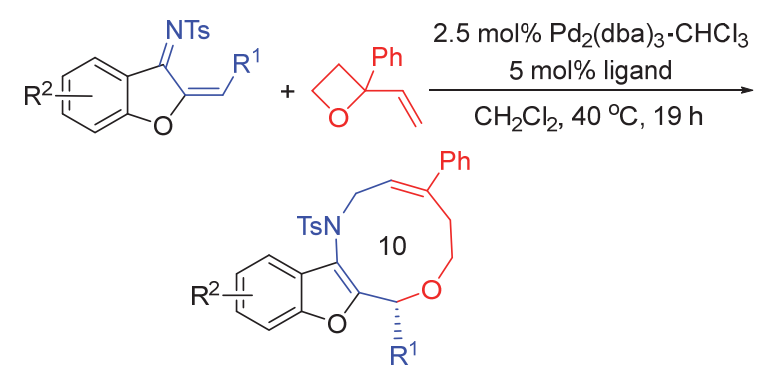

2019 年, 杜玮和陈应春课题组 ${ }^{[25]}$ 报道了以 MoritaBaylis-Hillman (MBH)醇与偶氮甲碱亚胺为底物, 在胺 与硫醇的双活化催化下，发生的 $[5+3]$ 环加成反应.<smiles>[R]C=C1Oc2ccccc2C1=[N]</smiles>

$\mathrm{MBH}$ 醇 38 和偶氮甲碱亚胺 39 反应，产生 $[5+3]$ 环加成 化合物 40.40 在 $\mathrm{H}_{2}$ 气氛下, 烯酮部位会完全氢化，同时 $\mathrm{N}-\mathrm{N}$ 键裂解，合成含有 8 元环的双环产物 41 (Scheme 16).
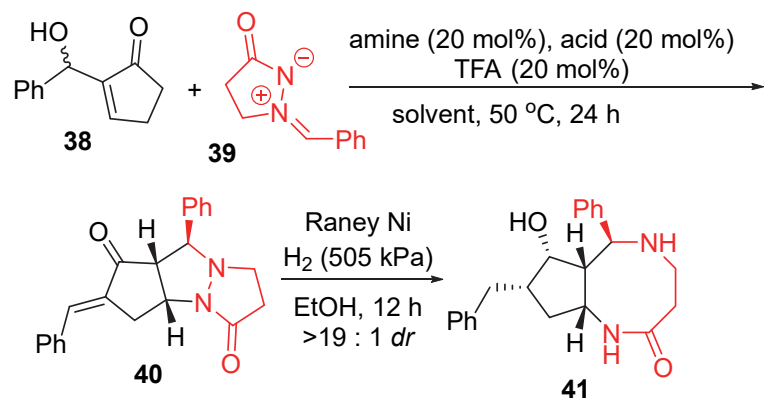

图式 16 胺与硫醇双活化催化的 $[5+3]$ 环加成反应 Scheme 16 [5+3] Cycloaddition catalyzed by double activation of amine and mercaptan

\section{4 基于自由基迁移的扩环反应}

2013 年, Tan 课题组 ${ }^{[26]}$ 报道了一种逐步氧化去芳构 化扩环反应(ODRE). 中间体 $\mathbf{J}$ 溶剂化形成稳定的环己 二酮中间体，该中间体会自发地进行扩环重新芳构化， 形成正离子化叔碳, 消除 E1 后生成含有 $8 \sim 11$ 元环的 43 (Scheme 17). 在此基础上, 2018 年, 该课题组 ${ }^{[27]}$ 报道 了一种新的氧化脱芳扩环反应(ODRE). 双环苯酚的底 物经过氧化去芳构化形成亲电环己二烯基阳离子中间体, 然后与侧链亲核试剂反应生成三环的环己二烯酮，该中 间体被 Brønsted 酸、Lewis 酸或三氟酸酐活化引起扩环, 同时苯酚环重新芳构化构建 8１1 元环(Scheme 18).
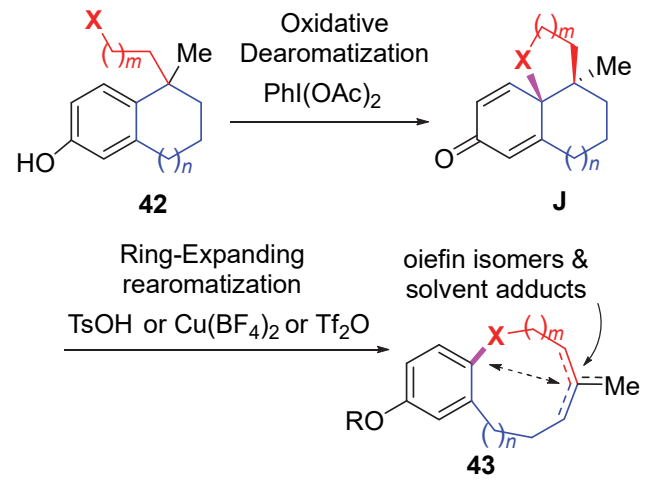

图式 17 逐步氧化去芳构化扩环反应

Scheme 17 Stepwise oxidative dearomatization-ring-expanding rearomatization 


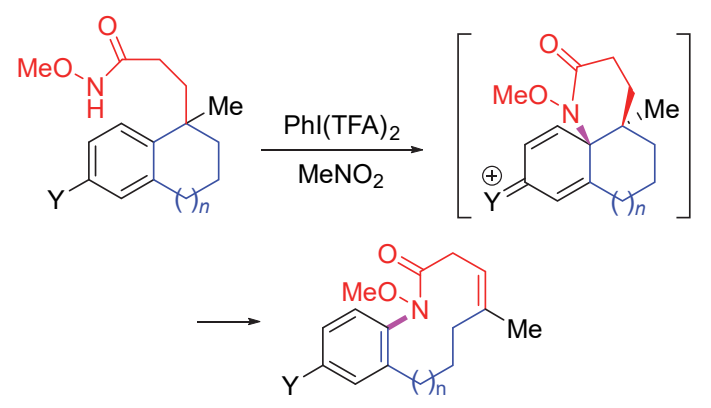

图式 18 ODRE 反应

Scheme 18 ODRE reaction

2016 年, Clayden 课题组 ${ }^{[28]}$ 报道了一种通过杂环前 体的 $n \rightarrow n+3$ 扩环合成中环苯并稠合的含氮杂环的反应 (Scheme 19). 该反应基于芳基环从 $\mathrm{N}$ 到 $\mathrm{C}$ 的立体定向迁 移, 44 中苄位上的 C 去质子化生成所示阴离子, 此类阴 离子会对 $N$-芳基取代基进行亲核攻击完成扩环生成含 有 $8 \sim 12$ 元环的 45 .

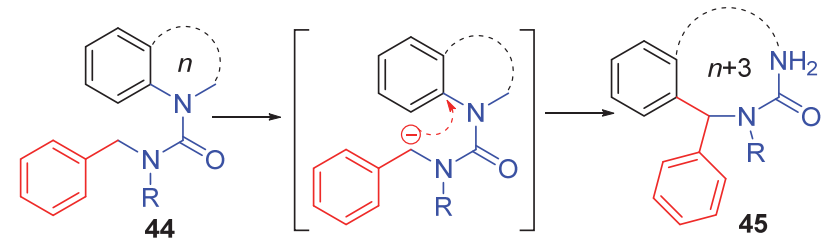

图式 $19 n+3$ 扩环反应

Scheme $19 n+3$ Ring expansion

2016 年, 刘心元课题组 ${ }^{[29]}$ 报道了由三氟甲基化、叠 氮化、磺酰化和全氟烷基化引发的 1,2-, 1,4-或 1,5-甲酰 基自由基迁移的策略，该策略成功开发了第一个自由基 烯烃 1,2-双功能化型的甲酰化反应或未活化的烯烃的二 氟甲基化反应, 从而得到多种 $\beta$-官能化的醛(Scheme 20). 在此基础上, 该策略还可以获得各种 9 11 元环, 以进一步转化为不同类型的环系统, 包括具有各种功能 的稠环和桥环. 该课题组还报道了以烯基环状 $\alpha$-差基酮
为底物，基于远程 1,4-或 1,5-羰基迁移的扩环反应(Eq. 4).<smiles>[R]C(=C)/C(=C\C(=O)O)C(=O)O</smiles>

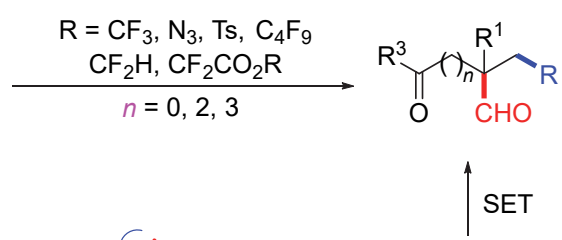

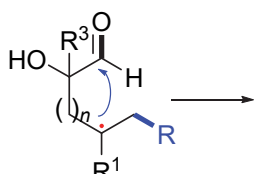

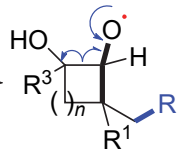

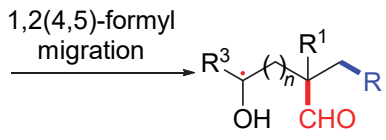

图式 20 烯烃的 1,2-甲酰基官能化及其合成应用 Scheme 20 1,2-Formylfunctionalization of alkenes and synthetic applications

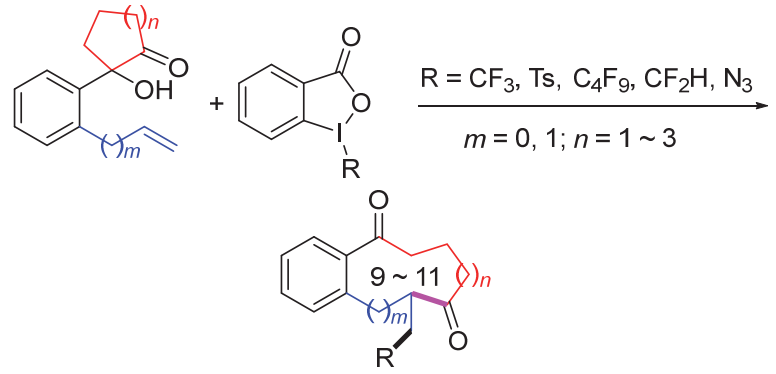

2016 年, 刘心元课题组 ${ }^{[30]}$ 报道了一种通过未活化 烯烃的自由基叠氮化、三氟甲基化、膦酰化、磺酰化或 全氟烷基化触发的 1,4-或 1,5-芳基迁移/环扩张反应。该 反应以易得的底物实现了 1,4-和 1,5-芳基迁移，可得到 的环尺寸范围较广, 自由基来源较多. 该反应在 46 和 47 的物质的量之比为 $1.0: 1.2$ 的条件下, 在 $\mathrm{CuCN}(10$ mol\%)和 EtOAc 中于 $60{ }^{\circ} \mathrm{C}$ 下反应 $12 \mathrm{~h}$ 得到含有 $8 \sim 11$ 或 14 元环的 48 (Scheme 21).

2017 年, 刘心元课题组 ${ }^{[31]}$ 首次报道了通过远程自 由基烯基迁移以及 $\mathrm{C}-\mathrm{C}$ 键的断裂构建含 8 14 元环的 烯烃分子的反应(Scheme 22). 该反应以 $\mathrm{CuCN}(10$

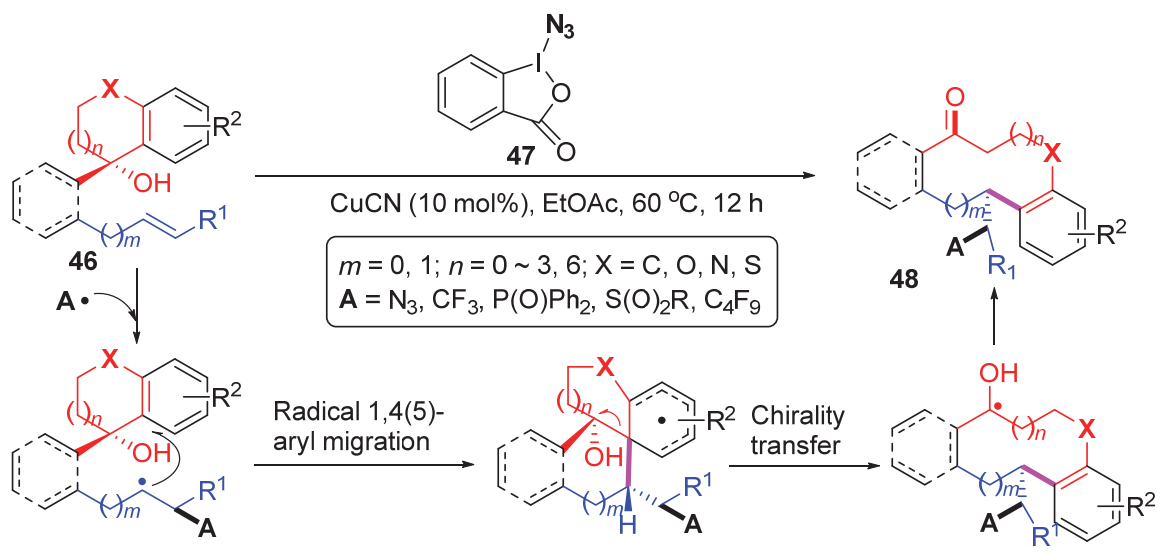

图式 21 1,4-或 1,5-芳基迁移/扩环反应

Scheme 21 1,4- or 1,5-Aryl migration/ring expansion 


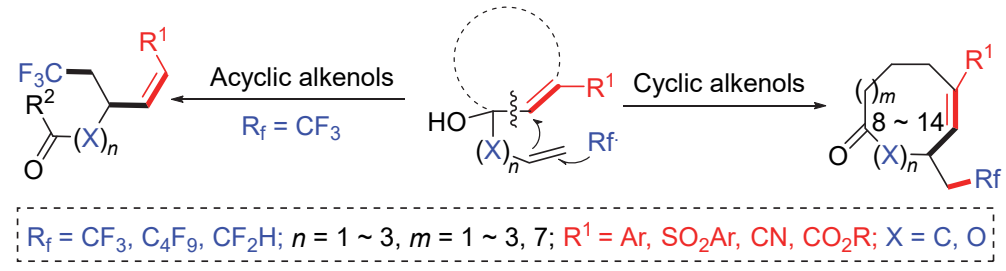

图式 22 远程乙烯基迁移和合成应用

Scheme 22 Remote radical vinyl migration and synthetic application

mol\%)为催化剂，1,4-二噁烷为溶剂，通过远程乙烯基迁 移的方法合成中/大环的氟代烷基化环状烯烃. 该课题 组提出远端乙烯基迁移的机理: 各种氟代烷基提供自由 基，选择性地攻击位阻较小的末端烯烃，从而得到过渡 态的烷基自由基 $\mathbf{K}$. 随后碳自由基进攻双键产生环化的 中间体 $\mathbf{K}$, 中间体 $\mathbf{L}$ 通过 $\beta$ 裂解可导致远端的自由基乙 烯基迁移/扩环形成中间体 $\mathbf{M}$, 进一步形成氟代烷基化 的含 $8 \sim 14$ 元环的烯烃(Scheme 23).
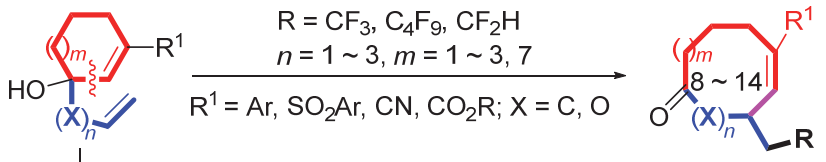

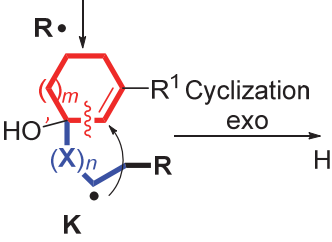
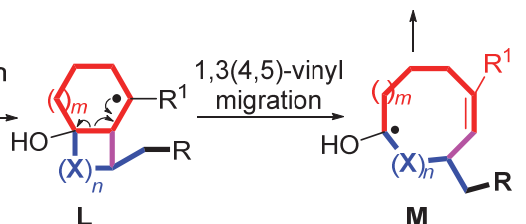

图式 23 远程乙烯基迁移机理

Scheme 23 Proposed mechanism for remote radical vinyl migration

Beckwith-Dowd 扩环反应 ${ }^{[32]}$ 是合成中大环化合物 的一种有效的方法. 2012 年, Lupton 课题组 ${ }^{[33]}$ 利用 Beckwith-Dowd 扩环级联反应制备了一系列螺环 $\gamma$-内 酯, 环酮内发生碳扩建, 然后进行 5-exo-trig 或 5-exo-dig 环化生成含有 $6 \sim 10$ 元环的 $\gamma$-内酯(Scheme 24). 2018 年, Itoh 课题组 ${ }^{[34]}$ 报道了由光诱导的 Beckwith-Dowd 扩环反 应. 在氮气氛围下, 用荧光灯照射 $20 \mathrm{~h}$, 以二甲基亚砜 (DMSO) 为溶剂, 将底物 49 与胺、红霉素 $\mathrm{B}(\mathrm{EB}) 、{ }^{i} \mathrm{Pr}_{2} \mathrm{NEt}$ 混合, 生成所需产物 50. 作者推断其反应机制为: $\mathrm{EB}^{2-}$ * 或 $\mathrm{EB}^{-*}$ 诱导单电子转移(SET), 导致 49 的 $\mathrm{C}-\mathrm{I}$ 键裂解, 产生的自由基 51 与酮反应, 环丙烷 C-C 键断裂完成扩 环生成环酩(Scheme 25). 2020 年, 朱晨课题组 ${ }^{[35]}$ 报道了 自由基介导环己酮和环戊酮非张力环的 $\mathrm{C}-\mathrm{C}$ 裂解合成 苯并呋喃和狮满酮的策略, 并揭示了不同于 BeckwithDowd 扩环反应中 $\mathrm{C}-\mathrm{C}$ 键选择性断裂的原因.

2018 年, 刘心元课题组 ${ }^{[36]}$ 报道了在光催化条件下 产生的自由基的(杂)芳基远程 $\mathrm{C} \rightarrow \mathrm{N}$ 迁移, 通过 $n+3$ 扩 环策略发展了由(杂)芳基环酮制备含有 $8 \sim 11$ 元环的内
酰

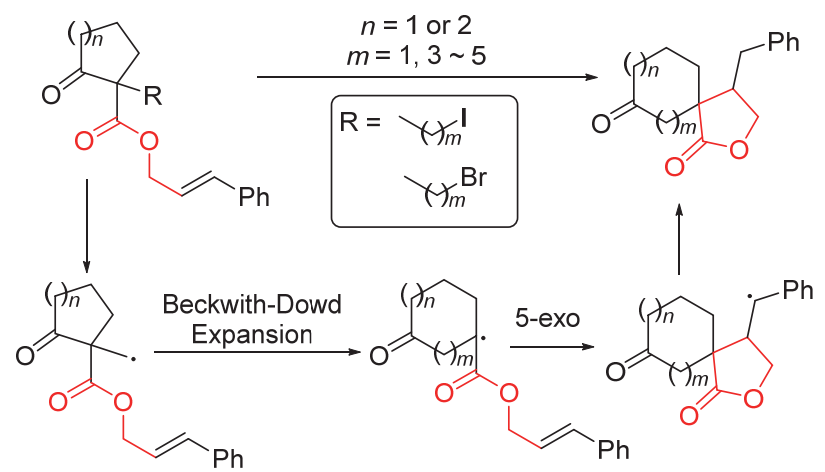

图式 24 Beckwith-Dowd 扩环级联反应

Scheme 24 Cascade Beckwith-Dowd ring expansion/ cyclization<smiles></smiles><smiles>O=C1CCCCC1C(=O)OCCO</smiles>

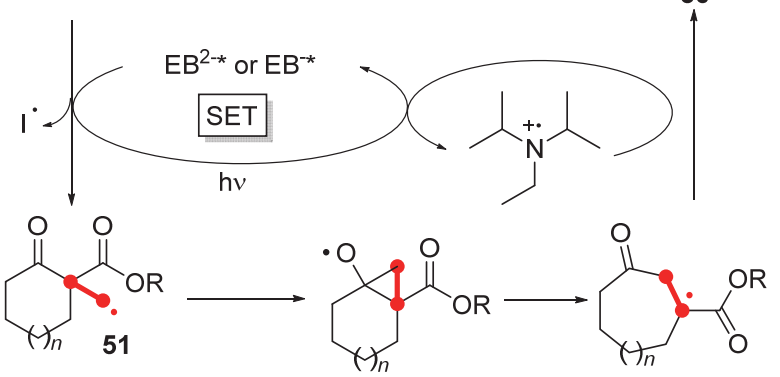

图式 25 Beckwith-Dowd 扩环

Scheme 25 Beckwith-Dowd ring expansion

环新方法(Scheme 26). 该方法以 $\mathrm{Ru}(\mathrm{bpy}){ }_{3} \mathrm{Cl}_{2} \bullet 6 \mathrm{H}_{2} \mathrm{O}(1.5$ mol\%)为光催化剂, BI-OAc (1.5 equiv.)为氧化剂, 1,2-二 氯乙烷(DCE)为溶剂，蓝色 LED 灯为光源，室温反应 $5 \mathrm{~h}$ 可以获得 9 元环产物. 该课题组提出反应的机理为: 激

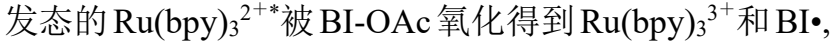
底物 52 被 $\mathrm{BI} ・$ 和羧酸氧化得到胺基自由基中间体 $\mathbf{N}$, 胺 基自由基进攻芳环得到中间体 $\mathbf{O}$, 并发生 $\mathrm{C}-\mathrm{C}$ 键断裂 得到中间体 $\mathbf{P}, \mathrm{Ru}(\mathrm{bpy})_{3}^{3+}$ 与中间体 $\mathbf{P}$ 发生单电子转移 得到目标物 53 .

2018 年, 刘心元课题组 ${ }^{[37]}$ 报道了通过三氟甲基自 


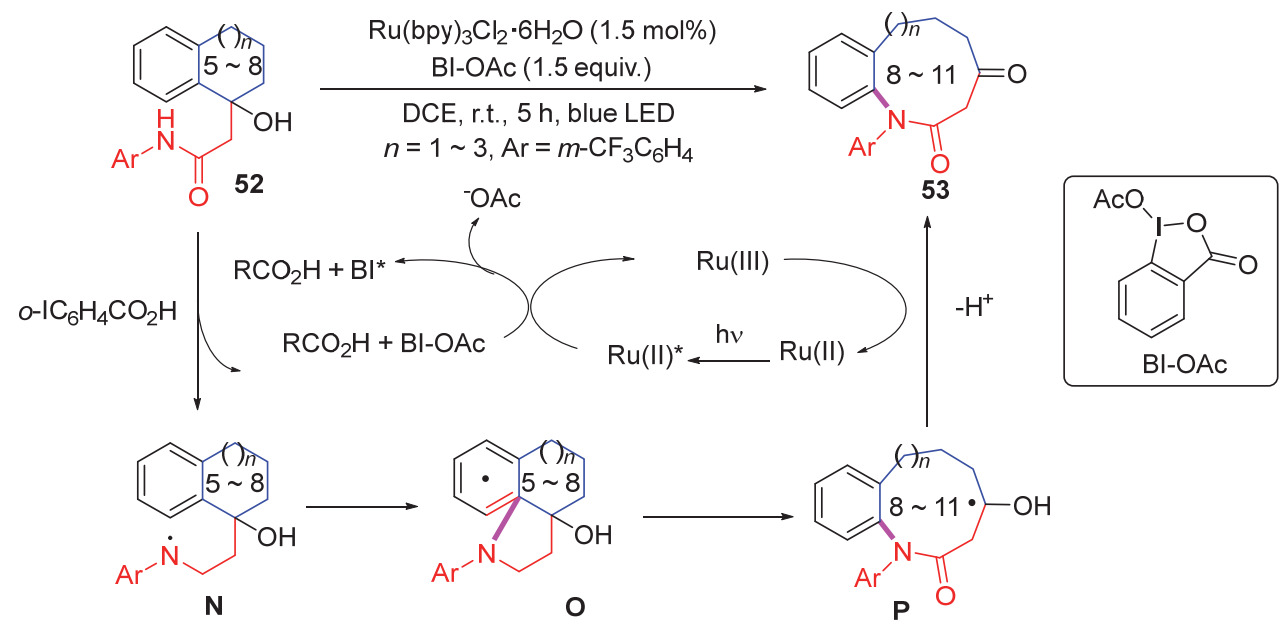

图式 26 酮合成中型内酰胺的扩环反应

Scheme 26 Ring-expansion strategies for synthesis of medium-sized lactams from ketones

由基诱导的分子内肟基的远程迁移，使肜基三氟甲基化 的有效策略(Eq. 5). 该策略可以用于合成中环: 通过分 子内自由基 1,4-和 1,5-肟醚转移来进行扩环的反应(Eq. 6). 该反应使用具有环戊酮肟基的底物 54 与 Togni’s 试 剂 55 在 $\mathrm{CuCN}$ 、室温条件下反应 $24 \mathrm{~h}$ 生成含有 $8 \sim 10$ 元环的肟 56.

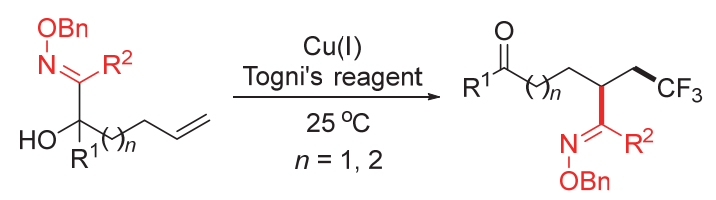<smiles>C=CCc1ccccc1C1(O)CCCC1=NOCc1ccccc1</smiles>

2019 年, Knowles 课题组 ${ }^{[38]}$ 报道了一种以异构环醇 为底物, Brønsted 碱为催化剂, 蓝色 LED 灯照射下, $\mathrm{O}$ $\mathrm{H}$ 键上发生质子偶合电子转移(proton-coupled electron transfer, PCET), 完成 $n+1$ 或 $n+2$ 扩环的反应. 该反应 的机理为: PCET 生成的烷氧基自由基会发生开环 $\beta$ 断 裂，生成 $\alpha, \beta$-不饱和酮和烷基; 烷基与形成的烯酮结合， 在碳环框架内形成一个新的 $\mathrm{C}-\mathrm{C}$ 键; 在 $n+2$ 扩环反应 的过程中, Ir(II)状态的光催化剂还原生成的 $\alpha$-酰基, 生 成烯醇化的中间体在 Brønsted 碱的共轭酸作用下质子 化并形成 $n+2$ 扩环产物. 过程中加入 $\mathrm{H}$ 原子供体即可 引发氢原子转移生成 $n+1$ 扩环产物(Scheme 27).

2020 年, 阮志雄课题组 ${ }^{[39]}$ 报道了一种通过 C-C 键 断裂的酰胺基迁移合成中环内酰胺的首个电化学合成 方法. 反应首先在阳极进行, 由 57 中的酰胺基 $\mathrm{N}-\mathrm{H}$ 键 氧化, 生成酰胺基中间体 $\mathbf{Q}$, 然后该中间体与芳基环进 行分子内环化，中间体 $\mathbf{S}$ 中形成新的 $\mathrm{C}-\mathrm{N}$ 键, $\mathrm{C}-\mathrm{C}$ 键 选择性断裂，形成中性的酮基自由基 $\mathbf{T}$. 最后，酮基自 由基 $\mathbf{T}$ 经过单电子氧化失去质子生成含有 $8 \sim 11$ 元环的 内酰胺 58 (Scheme 28).

\section{3 总结与展望}

以环化反应和扩环反应两方面为分类依据，简要地 总结了近 5 年以来中环大环的合成工作. 我们可以看到

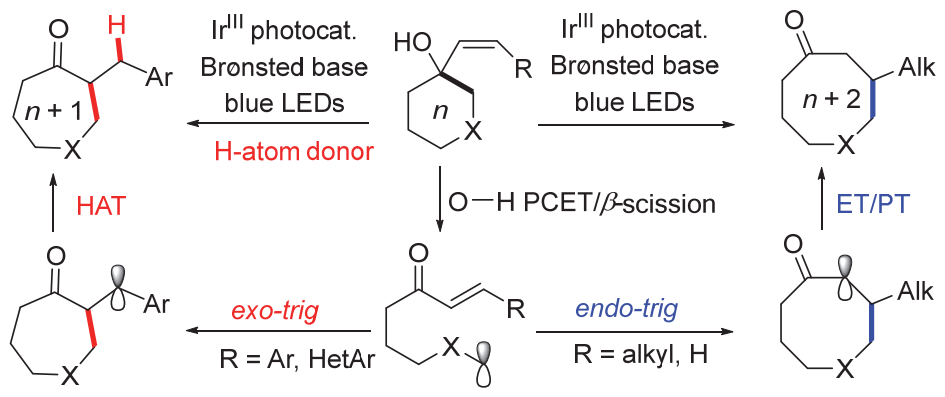

图式 27 质子偶合电子转移导致的扩环反应

Scheme 27 PCET-enabled ring-expansion 


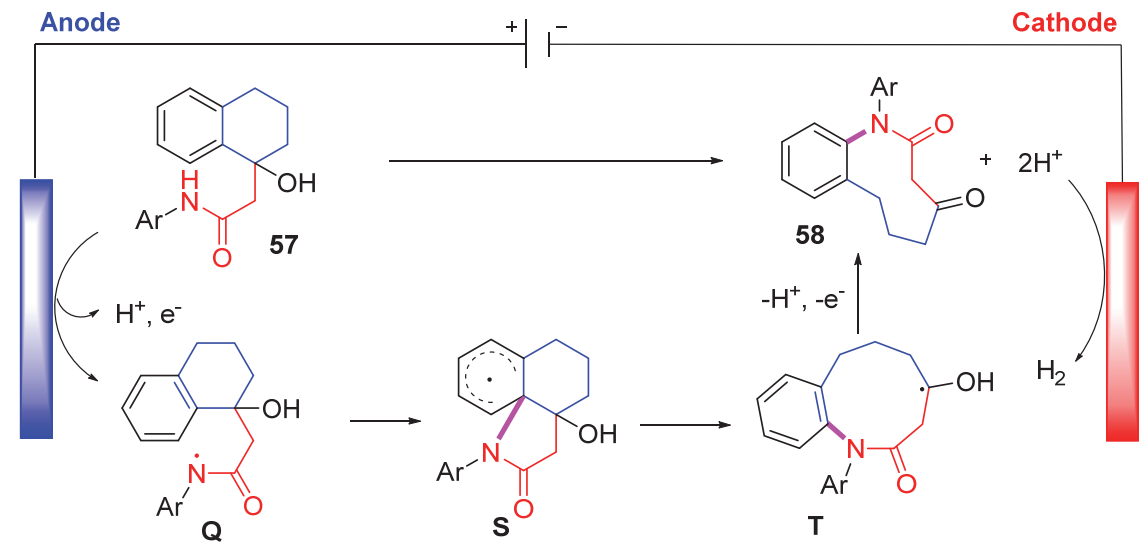

图式 28 通过 $\mathrm{C}-\mathrm{C}$ 裂解电化学合成内酰胺

Scheme 28 Electrochemical synthesis of lactams by $\mathrm{C}-\mathrm{C}$ cleavage

这两大类合成中环大环的方法各有优劣, 很多方法仍具 有催化剂价格昂贵、反应条件严格及底物制备困难等局 限. 因此, 简化与经济化实现中环大环合成仍是亟待解 决的问题. 同时我们也注意到新试剂、新反应与新策略 的发展一定程度上简化了中环大环合成路线, 特别是近 年来利用自由基迁移扩环方法合成中环大环化合物的 策略.

在未来, 对中环大环合成方法的探索应建立在清晰 正确的理论基础知识之上, 克服当前合成方法的局限 性，向高效、简便及环境友好的方向发展. 尤其是随着 新反应和新试剂的出现, 希望未来在多组分环化合成中 环大环策略上, 通过设计可控的串联反应, 能够由简单 分子精准合成特定环大小的复杂大环化合物; 在扩环策 略上通过预置官能团更加精巧地设计小环底物分子, 特 别是最近发展的环化一扩环反应策略, 不用分离出小环 中间体, 一步实现小环到大环的高效构建. 另外利用光 催化和电催化的绿色合成研究还较少, 因此, 期望未来 有更多的工作致力于使用绿色环保的方法构建中环大 环结构.

随着科学技术的进步以及研究人员的努力, 大环化 合物的合成在将来会有更实质性的进展, 并在药物合 成、有机化学和生命科学等重要领域拥有更加广阔的应 用前景.

\section{References}

[1] Hesse, M. Ring Enlargement in Organic Chemistry, Wiley-VCH, Weinheim, Germany, 1991.

[2] (a) Clarke, A. K.; Unsworth, W. P. Chem. Sci. 2020, 11, 2876. (b) Terrett, N. K. Drug Discovery Today: Technol. 2010, 7, e97. (c) Hussain, A.; Yousuf, S. K.; Mukherjee, D. RSC Adv. 2014, 4, 43241.

[3] Khan, A. R.; Parrish, J. C.; Fraser, M. E.; Smith, W. W.; Bartlett, P. A.; James, M. N. G. Biochemistry 1998, 37, 16839.

[4] Kwon, Y.-U.; Kodadek, T. Cell Chem. Biol. 2007, 14, 671.

[5] Maier, M. E. Angew. Chem., Int. Ed. 2000, 39, 2073.
[6] Zhao, Y.; Lv, Y.; Xia, W. Chem. Rec. 2018, 19, 424.

[7] Yet, L. Chem. Rev. 2000, 100, 2963.

[8] Vougioukalakis, G. C.; Grubbs, R. H. Chem. Rev. 2010, 110, 1746.

[9] (a) Beck, B.; Larbig, G.; Mejat, B.; Magnin-Lachaux, M.; Picard, A.; Herdtweck, E.; Dömling, A. Org. Lett. 2003, 5, 1047.

(b) Leon, F.; Rivera, D. G.; Wessjohann, L. A. J. Org. Chem. 2008, 73,1762 .

(c) Abdelraheem, E. M. M.; Kurpiewska, K.; Kalinowska-Tłuścik, J.; Dömling, A. J. Org. Chem. 2016, 81, 8789.

[10] Abdelraheem, E. M. M.; Madhavachary, R.; Rossetti, A.; Kurpiewska, K.; Kalinowska-Tłuścik, J.; Shaabani, S.; Dömling, A. Org. Lett. 2017, 19, 6176.

[11] Madhavachary, R.; Abdelraheem, E. M. M.; Rossetti, A.; TwardaClapa, A.; Musielak, B.; Kurpiewska, K.; Kalinowska-Tłuścik, J.; Holak, T. A.; Dömling, A. Angew. Chem., Int. Ed. 2017, 56, 10725.

[12] Zhou, B.; Zhang, Y.-Q.; Zhang, K.; Yang, M.-Y.; Chen, Y.-B.; Li, Y.; Peng, Q.; Zhu, S.-F.; Zhou, Q.-L.; Ye, L.-W. Nat. Commun. 2019, 10, 3234.

[13] Lawer, A.; Rossi-Ashton, J. A.; Stephens, T. C.; Challis, B. J.; Epton, R. G.; Lynam, J. M.; Unsworth, W. P. Angew. Chem., Int. Ed. 2019, 58,13942 .

[14] Ni, R.; Mitsuda, N.; Kashiwagi, T.; Igawa, K.; Tomooka, K. Angew. Chem., Int. Ed. 2015, 54, 1190.

[15] Mathiew, M.; Tan, J. K.; Chan, P. W. H. Angew. Chem., Int. Ed. 2018, 57, 14235.

[16] Kitsiou, C.; Hindes, J. J.; I'Anson, P.; Jackson, P.; Wilson, T. C.; Daly, E. K.; Felstead, H. R.; Hearnshaw, P.; Unsworth, W. P. Angew. Chem., Int. Ed. 2015, 54, 15794.

[17] Stephens, T. C.; Lodi, M.; Steer, A. M.; Lin, Y.; Gill, M. T.; Unsworth, W. P. Chem.-Eur. J. 2017, 23, 13314.

[18] Stephens, T. C.; Lawer, A.; French, T.; Unsworth, W. P. Chem.-Eur. J. 2018, 24, 13947

[19] Costil, R.; Lefebvre, Q.; Clayden, J. Angew. Chem., Int. Ed. 2017, 56,14602 .

[20] Zhao, W.; Qian, H.; Li, Z.; Sun, J. Angew. Chem., Int. Ed. 2015, 54, 10005.

[21] Zhou, Y.; Wei, Y. L.; Rodriguez, J.; Coquerel, Y. Angew. Chem., Int. Ed. 2019, 58, 456.

[22] Kasun, Z. A.; Geary, L. M.; Krische, M. J. Chem. Commun. 2014, 50,7545 .

[23] Yang, L. C.; Rong, Z. Q.; Wang, Y. N.; Tan, Z. Y.; Wang, M.; Zhao, Y. Angew. Chem., Int. Ed. 2017, 56, 2927.

[24] Wang, Y. N.; Yang, L. C.; Rong, Z. Q.; Liu, T. L.; Liu, R.; Zhao, Y. Angew. Chem., Int. Ed. 2018, 57, 1596.

[25] Yang, Q.-Q.; Yin, X.; He, X.-L.; Du, W.; Chen, Y.-C. ACS Catal. 2019, 9, 1258 .

[26] Bauer, R. A.; Wenderski, T. A.; Tan, D. S. Nat. Chem. Biol. 2013, 9, 21. 
[27] Guney, T.; Wenderski, T. A.; Boudreau, M. W.; Tan, D. S. Chem.Eur. J. 2018, 24, 13150.

[28] Hall, J. E.; Matlock, J. V.; Ward, J. W.; Gray, K. V.; Clayden, J. Angew. Chem., Int. Ed. 2016, 55, 11153.

[29] Li, Z. L.; Li, X. H.; Wang, N.; Yang, N. Y.; Liu, X. Y. Angew. Chem., Int. Ed. 2016, 55, 15100.

[30] Li, L.; Li, Z.-L.; Wang, F.-L.; Guo, Z.; Cheng, Y.-F.; Wang, N.; Dong, X.-W.; Fang, C.; Liu, J.; Hou, C.; Tan, B.; Liu, X.-Y. Nat. Commun. 2016, 7, 13852.

[31] Li, L.; Li, Z.-L.; Gu, Q.-S.; Wang, N.; Liu, X.-Y. Sci. Adv. 2017, 3, e1701487.

[32] (a) Dowd, P.; Choi, S. C. J. Am. Chem. Soc. 1987, 109, 3493.

(b) Beckwith, A. L. J.; O'Shea, D. M.; Westwood, S. W. J. Am. Chem. Soc. 1988, 110, 2565.
[33] Hierold, J.; Lupton, D. W. Org. Lett. 2012, 14, 3412.

[34] Deguchi, M.; Fujiya, A.; Yamaguchi, E.; Tada, N.; Uno, B.; Itoh, A. RSC Adv. 2018, 8, 15825.

[35] Wang, M.; Li, M.; Yang, S.; Xue, X.-S.; Wu, X.; Zhu, C. Nat. Commun. 2020, 11, 672.

[36] Wang, N.; Gu, Q.-S.; Li, Z.-L.; Li, Z.; Guo, Y.-L.; Guo, Z.; Liu, X.-Y. Angew. Chem. 2018, 57, 14225.

[37] Wang, N.; Wang, J.; Guo, Y. L.; Li, L.; Sun, Y.; Li, Z.; Zhang, H. X.; Guo, Z.; Li, Z. L.; Liu, X. Y. Chem. Commun. 2018, 54, 8885.

[38] Zhao, K.; Yamashita, K.; Carpenter, J. E.; Sherwood, T. C.; Ewing, W. R.; Cheng, P. T. W.; Knowles, R. R. J. Am. Chem. Soc. 2019, 141,8752 .

[39] Xu, Z.; Huang, Z.; Li, Y.; Kuniyil, R.; Zhang, C.; Ackermann, L.; Ruan, Z. Green Chem. 2020, 22, 1099.

(Zhao, C.) 